\title{
Selected papers from the 3rd European Conference on Microfluidics: $\mu$ Flu'12
}

\author{
Stéphane Colin · Juergen J. Brandner • \\ Gian Luca Morini · Bernd Michel
}

Published online: 30 January 2015

(C) Springer-Verlag Berlin Heidelberg 2015

The Third European Conference on Microfluidics ( $\mu$ Flu'12) was held in Heidelberg, Germany, in December 8-10, 2012, under the sponsorship of the Hydrotechnic Society of France (SHF), the Alma Mater Studiorum Università di Bologna, the University of Toulouse and the Karlsruhe Institute of Technology.

This event has gathered 252 participants from 33 countries. During three days, 174 communications have been presented in 16 thematic sessions including liquid microflows, gas microflows, microsensors and in-situ analytics, fluidic microactuators and micromixing, microfabrication techniques for microfluidic systems, convective micro heat transfer, micro chemical engineering, lab-on-a-chip, electrokinetic microflows, microdroplets management, microflows in biological systems and bioengineering, multi-phase flows

Stéphane Colin, Juergen J. Brandner and Gian Luca Morini are Co-Chairs of $\mu$ Flu' 12 and Guest Editors of Microsystem Technologies.

\section{S. Colin $(\bowtie)$}

Université de Toulouse, INSA, ICA (Institut Clément Ader), 135 Avenue de Rangueil, 31077 Toulouse, France

e-mail: stephane.colin@insa-toulouse.fr

\section{J. J. Brandner}

Karlsruhe Institute of Technology, Institute for Micro Process Engineering (IMVT), Campus North, Hermann-von-Helmholtz-Platz 1, 76344 Eggenstein-Leopoldshafen, Germany

e-mail: juergen.brandner@kit.edu

\section{G. L. Morini}

DIN, Alma Mater Studiorum Università di Bologna, 40136 Bologna, Italy

e-mail: gianluca.morini3@unibo.it

B. Michel

Microsystem Technologies, Berlin, Germany in microsystems, and microflow visualization. Two special sessions have been devoted to industrial applications of microfluidics and to nanofluidics.

This special issue of Microsystem Technologies focuses the attention of the readers on a selection of nine works representative of the research advances in microfluidics presented during the Conference.

Carrier et al. experimentally analyzed the mechanism of satellite droplets formation in the wake of the main droplet generated in a flow-focusing junction, with Newtonian or non-Newtonian liquids. By confocal laser scanning microscopy, Ben Hassan et al. experimentally observed the fouling of a filter due to the accumulation of microparticles on a membrane surface, and developed a numerical model able to predict the cake growth during the filtration. The paper of Kong and Nguyen reports a technique for the fabrication of three-dimensional multilayer liquid-metal microcoils by lamination of dry adhesive films; these microcoils can be used for magnetic resonance relaxometry (MRR) measurement in a lab-ona-chip platform. Samouda et al. developed a new setup for the experimental investigation of gaseous microflows by molecular tagging velocimetry-MTV-and discussed its possible use to obtain a direct measurement of velocity slip at the walls in rarefied regimes. Cao et al. designed different tools for microfluidic droplet processing, including an automated "gradient take-up tool" for the generation of segment sequences with gradually changing composition of the individual droplets. Wibel et al. studied a novel microstructured evaporator geometry consisting of curved, instead of straight, microchannels and demonstrated that strong centrifugal forces led to an enhancement of the evaporation efficiency. Calaon et al. evaluated the capability of producing with high volume lab-on-chip devices through injection molding. 
Preparation of master geometries was made in a $\mathrm{Si}$ wafer using e-beam lithography and reactive ion etching. Subsequent nickel electroplating was employed to replicate the obtained geometries on the tool, which was used to mold on transparent polymer substrates the functional structures. Hemmilä et al. proposed a process that can be applied to integrate a microfluidic sample delivery system onto different silicon nanowire-based biosensors. Finally, Fürjes et al. designed a robust and cost-effective microfluidic part of an optical biosensor in PDMS, including sample preparation functions, by using SU-8 moulding replica.

We would like to thank the authors for presenting their works at the Conference and in this special issue. We would also like to extend our thanks to all the members of the Scientific Committee of the Conference for the accurate review process of each paper before the presentation to the Conference and, after the Conference, for the selection of the papers for the special issues linked to the Conference.

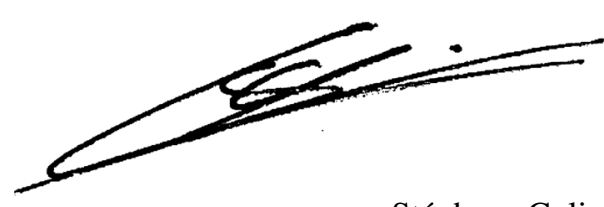

Stéphane Colin
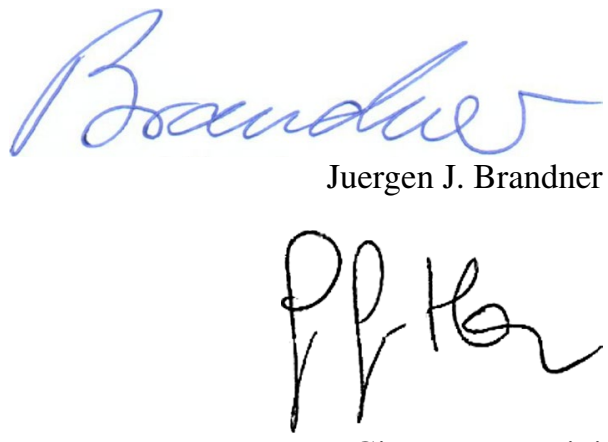

Gian Luca Morini

B. Mild

Bernd Michel 